\title{
DIGITAL SUPPORT FOR A HUB-AND-SPOKE DISEASE NETWORK
}

\author{
Paolo Locatelli ${ }^{1}$, Federica Cirilli $^{1}$, Eliana Panno ${ }^{1}$, Salvatore Provenzano ${ }^{2}$, Roberta Sanfilippo ${ }^{2}$ \\ and Paolo G. Casali ${ }^{2-3}$ \\ ${ }^{1}$ Fondazione Politecnico di Milano, Italy \\ ${ }^{2}$ Medical Oncology Unit 2, Fondazione IRCCS Istituto Nazionale dei Tumori di Milano, Italy \\ ${ }^{3}$ Department of Oncology and Haemato-Oncology, University of Milan, Italy
}

\begin{abstract}
A disease network is a collaborative healthcare network focusing on a single disease, or a group of diseases, whether professional or formalized. A disease network may follow a "hub and spoke" model. Providing these networks with an efficient digital support poses some challenges. This paper describes the methodological approach to design the information system supporting such a disease network. After identifying a general model with network features, a use case related to the Italian Rare Cancer Network is reported.
\end{abstract}

\section{KEYWORDS}

Rare Cancer, Collaborative Networks, Digital Healthcare, Teleconsultation

\section{INTRODUCTION}

A collaborative healthcare network is an alliance of professionals and/or institutions to achieve common or compatible goals by sharing their expertise and/or resources. A network may behave as a professional network, being a flexible alliance of health professionals, or may behave as a formal network of healthcare institutions. In general, the goal is to provide patients with fair and equal opportunities to access healthcare. Health networks often focus on a single disease, or a group of diseases (Morando, 2014; Camarinha-Matos et al., 2015). Networks must be served by an information system and thus require a strong digital support (Camarinha-Matos et al., 2008)0.

An area in which the effectiveness and the efficiency of health interventions can be substantially improved by networking has to do with rare diseases, including rare cancers. Rare cancers are defined by an incidence of $\leq 6 / 100,000 /$ year. They include 10 families of rare adult solid cancers, all childhood cancers and a group of haematological neoplasms. Overall, they represent more than $20 \%$ of all new cancer cases. Networking is largely seen as a key factor to improve effectiveness, limit health migration and reduce costs (both health and social costs). In the European Union (EU), the European Reference Networks (ERNs) have the task of tackling complex and rare diseases. They are mainly peer-to-peer networks. However, they should cooperate with national or regional networks, generally conceived as hub-and-spoke networks. One such network is the Italian Network on Rare Cancers, "Rete Nazionale Tumori Rari" (RNTR) (Casali, 2019).

The project of RNTR was approved in 2017 by the Italian government and regions, building on professional networks developed in the previous decades in Italy in the areas of rare adult solid cancers, childhood cancers and haematological neoplasms. Thus, the network is conceived as a formalized networks of healthcare institutions, whether hubs or spokes, supported by three professional networks, each covering one of those main groups of rare cancers. The network should help rationalize patient referral from throughout Italy towards its centers. Then, each professional network should coordinate a system of teleconsultations by which hubs share their expertise and resources with spokes (being thus named, respectively, "Providers" and "Users") (Locatelli et al., 2019; Elrod \& Fortenberry, 2017). 
This paper is meant to describe first the methodological approach to design the information system supporting such a disease network and secondly the general requirements of the information system identified following this approach. Then, starting from this general model, functional features which are more specific of this Italian disease network in the area of rare cancers are defined.

In literature, other similar works on digital support for collaborative networks have been reported (Costa et al., 2009; Noran, 2009); however, they focused on network decision support systems, not on systems supporting the real network collaboration. Other works focused on describing in detail methodologies and architectural models for planning, designing and implementing generic health information systems (Buranarach et al., 2011; Botheju, 2018). Moreover, in other papers (Thimm \& Rasmussen, 2010; Lenz \& Reichert, 2005), a tool for a collaborative network has been designed; however, a clear and general methodological approach on how the technical solution was achieved is not defined.

\section{THE METHODOLOGICAL APPROACH AND THE APPLICATION MODEL}

According to the Business Process Reengineering (BPR) methodology (Hammer \& Champy, 1993)0, a structured approach is described in Figure 1.

AS-IS process and digital support (if present) analysis. The first step consists in the analysis of the processes and, if present, the digital tools through which hubs and spokes collaborate. The team dealing with the digital support design needs to learn in detail the collaboration process.

Processes mapping, with related digital support (if present). The second step is mapping the collaboration process between hubs and spokes with the related digital tools, if present. This step allows the team to catch a rough summary of its actual functioning.

Identification of critical points and possible improvements. The third step consists in identifying weak points and critical issues within the current process, in order to define organizational, as well as technological, improvements.

Definition of the network needs. This step is aimed to define the network needs, including digital support ones, according to the process mapping made in the second step and to the weak points within the process identified in the previous step. The needs have to be defined in terms of organizational improvements and functionalities of the digital solution, in order to effectively support the collaboration within the disease network.

Design of the digital support. The final step is aimed to design the system specific functionalities with the technological provider in charge of the new digital support development. It is important to gain an alignment with the digital support needs and the developed functionalities.

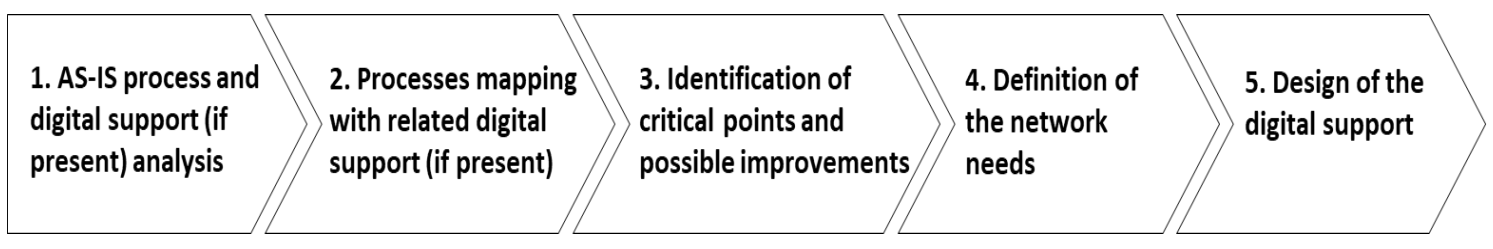

Figure 1. Methodological approach to design the digital support for a disease network

Following this approach, it is possible to generalize some aspects to any healthcare disease network. 


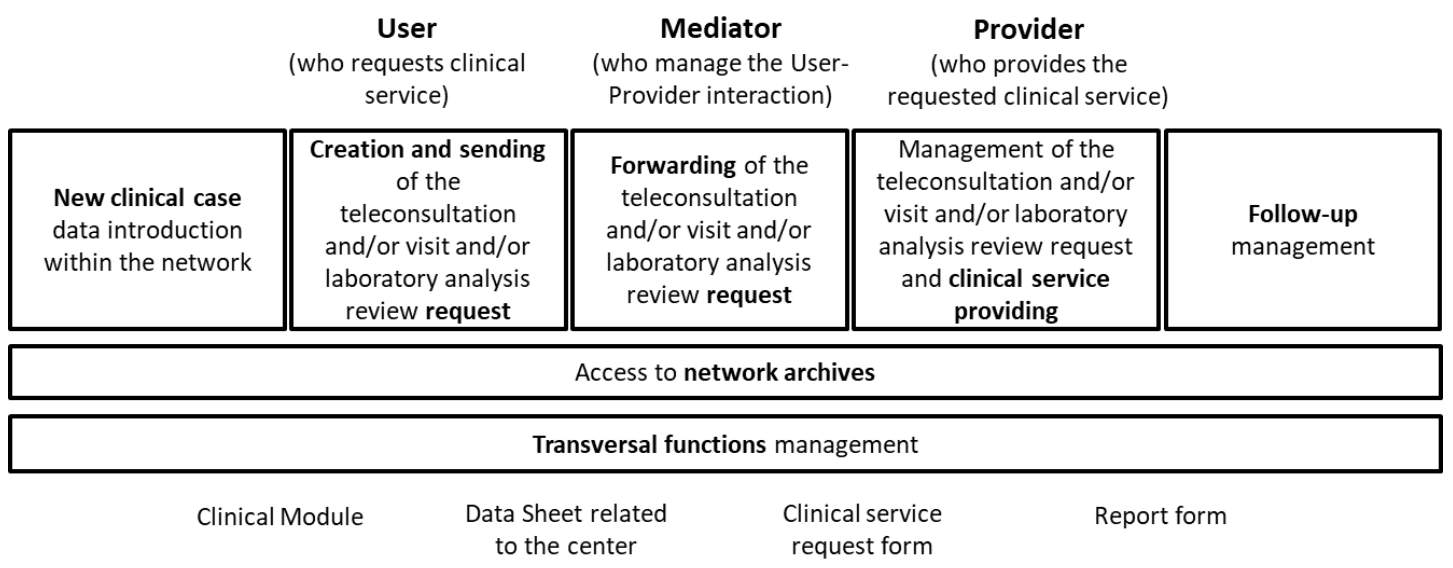

Figure 2. Schematic representation of the disease network features

Figure 2 shows a schematic representation of the disease network features, in terms of network users, system macro-functionalities and digital available forms.

Firstly, an information system supporting a disease network should ensure a User to submit a new clinical case, through the Clinical Module filling. Then, the User should be able to create and send a teleconsultation and/or visit request, using the appropriate clinical service request form. Moreover, he should be responsible for the follow-up by updating patient data within the Clinical Module. The User should also be authorized to access to the network archives, to search clinical cases for care purposes and to generate the teleconsultation report in a pdf format, possibly to be uploaded onto an online patient record. The system should allow the Mediator to manage the User-Provider interactions by automatically forwarding the clinical service request form, with the teleconsultation and/or visit request, to an adequate Provider within the network. On the other hand, the digital solution should support the Provider in managing the Data Sheet related to the centre availability and specialization, in visualizing the teleconsultation and/or visit and/or laboratory analysis request - with the possibility to request more details/further clarifications - and in providing the final report. Finally, more general and transversal system functionalities are essential to check the compliance with clinical practice guidelines, to assess the network efficiency through quality questionnaires for clinicians/patients and periodic monitoring of indicators.

Within an ongoing project, funded by the Italian Ministry of Health, "Italian Rare Cancer Network - Process Monitoring and System Impact Assessment", the methodological approach above-described was applied to design the new digital support for RNTR. The specific results are summarized in the next paragraph.

\section{DESIGNING A DIGITAL SUPPORT FOR THE ITALIAN RARE CANCER NETWORK}

Following the methodological approach, an analysis of the current management processes of patients in the disease network, building on the ongoing experience of the professional network in place as from 1997, was carried out. When the analysis was performed, the professional network already used a digital support. According to the methodological approach described above, we analysed this system, obsolete though it was, and we mapped the analysed processes. Then, according to the third and the fourth steps of the approach, we identified weak points and possible organizational/digital improvements and defined the network needs.

The Italian Rare Cancer Network includes different clinical centres with two main roles: Providers, who are the experts in a given rare cancer; Users, who request clinical services (e.g. teleconsultations) to the Providers; Mediators, who manage Provider-User interactions.

First, the patient is seen by the nearest local cancer centre. If this is a Provider, the patient will be managed at the centre, his/her case will be recorded in the information system, while only exceptionally will his/her case be shared with another Provider (e.g. the Provider does not own all clinical facilities needed to treat the patient). If the centre is a User, the oncologist can decide to request a teleconsultation, including a 
pathologic review, and/or a visit. In this phase, the digital support should allow the User to introduce and manage new clinical case data, through: automatic creation of a unique identification code, associated with the patient's name and surname; patient's signed consent archiving; patient's personal data entry; easy data entry of clinical information; reports and images loading, with data for the biological sample traceability. Moreover, the digital system should allow the User to create and send a teleconsultation request, filling the: teleconsultation request form, with the diagnostic question object, or the histological review request form.

Then, the Mediator makes sure that an appropriate Provider is involved and periodically checks the timeliness of interactions. A big challenge is to make such a function as much automatic as possible. For this reason, the system should allow the Mediator to manage and forward the teleconsultation and/or visit request, through automatic assignment of the case to the Provider and automatic monitoring of the User-Provider interaction time. Moreover, the system should send periodic alerts to the Provider.

Once the Provider is in charge of the patient, it can ask for further clinical information (e.g. clarifications on patient history, exams, etc.). It is very important to make sure that this interaction process between Users and Providers is allowed by the information system to proceed as swiftly as possible. In this context, the digital support should ensure the Provider to manage the received teleconsultation request and provide the service, through: visualization of the teleconsultation request and sending the reply ('Request accepted') to the Provider; request form filling asking for more details/further clarifications; visualization of the clinical data of the patient, with the possibility to integrate it (the system should guarantee the changes traceability); reports and images uploading (in the case of visit providing); teleconsultation report filling and sending, with notification of the adherence between the therapeutic path and the updated clinical guidelines.

Once received the Provider consultation, the system should guarantee the User to manage the follow-up through: visualization of the Provider's teleconsultation report; patient's clinical outcome data updating; generation of the teleconsultation report in the pdf format.

In general, both User and Provider should be able to access to the network archives searching for anonymized cases (in case of external patients) or searching for specific by using identification code or patient's name-surname (in case of internal patients). Finally, the system should allow the management of other functionalities, such as: visualization of updated clinical guidelines; questionnaires filling for clinicians or patients and some indicators monitoring, in order to verify the network efficiency and impacts.

In defining the solution development, the compliance with non-functional requirements, such as data accessibility, accuracy, availability and privacy, must be guaranteed.

We shared these requirements with the technological provider, and we are currently dealing with the final step of the methodological approach. Once completed this step and when the new digital support is developed, the project will test the system and a first set of data on the application will be available at the end of the project (September 2021).

\section{CONCLUSIONS}

In this paper we described first the methodological approach to designing the information system supporting such a hub-and-spoke disease network and secondly the general requirements of the information system identified following this approach. Then, starting from this general model, functional features of the Italian RNTR digital support are defined.

In order to assess the impacts of applying the network model and its new digital system, an appropriateness indicators panel has to be defined (Locatelli et al., 2014). Starting from the process analysis, supported by a literature search (Italian Ministry of Health, 2014; Frey, 2014), we identified a panel of indicators designed to monitor the appropriateness of a generic hub-and-spoke disease network:

Effectiveness indicators: managed network volumes (such as total number of cases introduced in the network by all Users or total number of consultations provided by a specific Provider); performance of the network (such as the average consultation provision time); clinical appropriateness (such as survival rate); healthcare migration; patients' and professionals' satisfaction.

Efficiency indicators:_social costs for healthcare migration; social costs for psychological assistance to the patient; healthcare costs for clinical inappropriateness (such as cost for inappropriate surgery).

These indicators will be monitored specifically for the RNTR as the project progresses. 


\section{ACKNOWLEDGEMENT}

The authors would like to thank the project partners: Fondazione IRCCS Istituto Nazionale Tumori di Milano, Istituto Europeo di Oncologia and Fondazione Politecnico di Milano. This work was supported by the Italian Ministry of Health (Grant No. RF-2016-02363386 to P.G.C.).

\section{REFERENCES}

Botheju D., "Smart eHealth" - A Holistic Approach to Implementing an Integrated Healthcare Information System (HIS) in Sri Lanka, 2018.

Buranarach M. et al., Design and Implementation of an Ontology-Based Clinical Reminder System to Support Chronic Disease Healthcare, IEICE Transactions on Information and Systems, (2011):432-439.

Camarinha-Matos L. M. et al., Classes of collaborative networks, Enciclopedia of networked and virtual organizations, (2008):193-198.

Camarinha-Matos L. M. et al., Risks and Resilience of Collaborative Networks, $16^{\text {th }}$ IFIP WG 5.5 Working Conference on Virtual Enterprises 2015.

Casali P.G., Trama A (eds). Rare Cancers Agenda 2030. EU Joint Action on Rare Cancers, 2019.

Costa R. et al., VirtualECare: Group Support in Collaborative Networks Organizations for Digital Homecare, in Handbook of Digital Homecare, Springer, ISBN: 978-3-642-01386-7, (2009):151-178.

Elrod J. K. \& Fortenberry J. L., The hub-and-spoke organization design revisited: a lifeline for rural hospitals, BMC Health Services Research (2017): 795.

Frey S., The economic burden of schizophrenia in Germany: A population-based retrospective cohort study using genetic matching, European Psychiatry, (2014):479-489.

Hammer M. \& Champy J., Reengineering the corporation: a manifesto for business revolution, Harper Business, New York, (1993).

Italian Ministry of Health, Telemedicine - national guidelines, 2014.

Lenz R. \& Reichert M., IT Support for Healthcare Processes, Business Process Management: Proceedings of the 3rd International Conference, Nancy, France (2005):354-363.

Locatelli P. et al. The role of the ICT in the process monitoring and system impact assessment of rare disease collaborative networks, Multi Conference on Computer Science and Information Systems, MCCSIS 2019 Proceedings of the International Conference on e-Health.

Locatelli P. et al., Effectively introducing mobile solutions in hospitals: The importance of process perspective and KPIs, Proceedings - IEEE Symposium on Computer-Based Medical Systems, (2014): 417-420.

Morando V., Cancer network configurations and regional health systems governance: a European comparison, Transatlantic Dialogue 2014 "From Public Administration to XXI Century Collaborative Administration: The role of public networks".

Noran O., A decision support framework for collaborative networks, International Journal of Production Research, vol. 47, no. 17, (2009):4813-4832.

Thimm H. \& Rasmussen K. B., Information Support Services for Intermediation Tasks of Collaborative Networks, Journal of Universal Computer Science, vol. 16, no. 13, (2010):1776-1800. 